\title{
Molecular mechanisms and potential prognostic effects of REST and REST4 in glioma (Review)
}

\author{
CUILIN LI ${ }^{1,2}$, ZHIFEI WANG ${ }^{3}$, XINYUE TANG ${ }^{1,2}$, LIU ZENG $^{1,2}$, XITANG FAN $^{3}$ and ZHI LI ${ }^{1,2}$ \\ ${ }^{1}$ Department of Clinical Pharmacology, Xiangya Hospital, Central South University, Changsha, Hunan 410008; \\ ${ }^{2}$ Institute of Clinical Pharmacology, Central South University and Hunan Key Laboratory of Pharmacogenetics, \\ Changsha, Hunan 410078; ${ }^{3}$ Department of Neurosurgery, Third Xiangya Hospital of \\ Central South University, Changsha, Hunan 410013, P.R. China
}

Received September 20, 2016; Accepted May 24, 2017

DOI: $10.3892 / \mathrm{mmr} .2017 .7071$

\begin{abstract}
Glioma refers to a tumor of the brain and central nervous system, which is characterized by high incidence, high mortality and high recurrence rate. Although the association between glioma and the repressor element silencing transcription factor (REST) has been reported by numerous studies, the complicated regulatory mechanisms underlying REST remain unknown. REST is a transcriptional repressor that undergoes alternative splicing to produce splicing variants when transcribed. Previous studies have demonstrated that alternative splicing may serve a role in the outcome of glioma. The present review discussed the mutual relationship among REST, REST4 and glioma. It was concluded that increased REST expression in glioma may be associated with poor prognosis; and REST4, an AS variant of REST, also functions to regulate glioma by suppressing REST. In addition, the present review discussed the regulation of REST and its target genes in glioma, and identified factors that induce REST alternative splicing, particularly in glioma. These findings suggest that REST may be considered a prognostic factor, which can be predictive of patient outcome.
\end{abstract}

\section{Contents}

1. Introduction

2. Repressor element silencing transcription factor

3. REST in glioma: Poor prognosis

4. Regulation and function of REST4

5. Regulation of REST expression in glioma

6. Gene regulation by REST in glioma

7. Conclusion

Correspondence to: Professor Zhi Li, Department of Clinical Pharmacology, Xiangya Hospital, Central South University, 110 Xiangya Road, Changsha, Hunan 410008, P.R. China E-mail: lizhi489@163.com

Key words: REST, REST4, glioma, molecular mechanisms, alternative splicing, prognostic factor

\section{Introduction}

Glioma, which refers to a tumor of the brain and central nervous system, accounts for $27 \%$ of all brain and CNS tumors, and $80 \%$ of malignant brain and CNS tumors diagnosed in the United States (1). The World Health Organization (WHO) classifies glioma into numerous histological subtypes; anaplastic astrocytoma (WHO grade III) and glioblastoma (WHO grade IV) are the most common. Due to the high mortality, high recurrence and low survival rates associated with this malignancy, the median overall survival time is $<1$ year (2). At present, radiotherapy, plus concomitant and adjuvant chemotherapy (temozolomide), following surgical resection represents the recommended therapeutic strategy for the treatment of newly diagnosed glioma (3). However, these cytotoxic therapies exhibit little improvement on overall survival, and are associated with severe toxic side effects. Therefore, further understanding the pathogenesis of glioma, and identifying novel strategies for the treatment of glioma, is required.

\section{Repressor element silencing transcription factor}

Molecular markers associated with the proliferation, apoptosis, invasion and metastasis of glioma have previously been reported. The present review aimed to evaluate the role of the transcriptional repressor, repressor element silencing transcription factor (REST). REST, which is also known as neuron-restrictive silencer factor (NRSF), is located at the $4 \mathrm{q} 12$ human chromosomal region, and is comprised of 4 exons and 3 introns. The full length of the REST gene is $24 \mathrm{~kb}$. REST is widely expressed in various types of cancer, including glioma, lung cancer (4) and breast cancer (5). Furthermore, the expression levels of REST were demonstrated to increase with age, and elderly people possess higher REST expression compared with younger individuals $(6,7)$. Regardless of age, the expression of REST may also be upregulated when neural cells become malignant $(8,9)$. In addition, a previous study identified a positive correlation between the expression of REST and the malignant degree of glioma (10).

The protein size of REST is 1,097 amino acids. As a transcriptional repressor, REST may inhibit its target 
genes by binding to the neuron-restrictive silencer element (NRSE) $(11,12)$. By connecting with two distinct corepressors, mSin 3 and CoREST, REST is able to regulate the expression of neuronal genes, via the recruitment of histone deacetylase complex (HDAC) to the promoters of REST-regulated genes in neuronal and non-neuronal cells $(13,14)$. In addition, REST has been demonstrated to act as a negative regulator of genes associated with numerous aspects of neuronal function, including neurogenesis, neural differentiation and preserving the specific neural phenotype (15-17). Furthermore, REST has been implicated to serve various roles in numerous cellular environments in the nervous system (18).

\section{REST in glioma: Poor prognosis}

In glioma, REST acts as an oncogene, which promotes proliferation and invasion of glioma cells. A previous study of 21 medulloblastoma tumor specimens demonstrated that all tumor specimens expressed higher levels of REST compared with the adjacent normal cerebellum tissue sections (6 strongly and 11 weakly) (19). Furthermore, when treated with REST-VP16, which is a competitor of endogenous REST/NRSF for DNA binding, the potential of REST intracranial tumorigenicity was suppressed and the growth of established tumors in nude mice was inhibited (19). These results indicated that overexpression of REST may contribute to medulloblastoma tumorigenesis and accelerate the proliferation of tumor cells. Furthermore, Blom et al (20) further hypothesized that REST may not directly influence glioma tumorigenesis but contribute to tumor development beyond the DNA level. The mRNA expression levels of REST were increased 2-5-fold in glioma tissue compared with in normal cerebral cortex tissues. Notably, when REST expression was knocked out in mice, the apoptotic and neuronal differentiation programs of malignant glioblastoma multiforme cell-derived xenograft tumors were activated (9). Taken together, these data suggested that REST may promote glioma development and is elevated in glioma compared with adjacent normal tissues. Furthermore, the mRNA expression levels of REST were significantly increased in grade III-IV glioma compared with in grade I-II glioma (10), thus suggesting that the expression of REST is positively correlated with the degree of glioma malignancy. However, low expression of REST was also detected in mouse neuroblastoma cell lines, such as NS20Y $(6,21,22)$. Due to this discrepancy, further research is required to validate the expression levels of REST in various species.

Due to the aforementioned expression profiles of REST in glioma, the present review hypothesized that there may be an association between REST expression levels and the prognosis of patients with glioma. Numerous studies have demonstrated that high REST expression is associated with poor prognosis in glioma. Wagoner and Roopra reported that patients with 'REST enhanced malignancies' (REM) tumors exhibited a significantly more aggressive disease course compared with patients with non-REM tumors (23), thus suggesting that enhanced REST may induce a more aggressive disease, and patients who express lower REST may have a better outcome. To verify these findings, a mouse xenograft experiment was conducted, which demonstrated that the injection of 'high REST' glioma cells into mice resulted in reduced survival compared with mice injected with 'low REST' glioma cells (8). In addition, elevated REST levels were associated with poor overall and event free survival in human patients with medulloblastoma (24). A further study in Chinese patients with neuroblastoma demonstrated that patients with late stage (grade IV) glioma who had higher REST activity were associated with poor survival compared with those in early stages (grades I and III combined) (25). Based on these experimental results, it is indicated that patients with glioma and high REST expression may have a poor prognosis.

\section{Regulation and function of REST4}

Alternative splicing of REST. It has been reported that $>90 \%$ of human genes, including phosphatase and tensin homolog, p53 (26), neurofibromatosis type I (27), and ATP binding cassette subfamily C member 1 (28), undergo alternative splicing, which contributes to the diversity of the transcriptome and proteome (29-31); there is no exception for REST. Several splicing variants of REST, including REST, REST1, REST4 and REST5 (Fig. 1), have been identified. However, among these variants, only REST4 and REST5 have been detected in neuronal tissues (32). REST4, with the insertion of exon $\mathrm{N}$ between exon III and IV of the REST gene, results in the early termination of translation, and has been reported to include the $\mathrm{N}$-terminal repression domain and number 1-5 zinc finger motifs, whereas REST5 lacks the number 5 zinc finger motif $(32,33)$. In addition, it has been demonstrated that the number 5 zinc finger motif is of crucial importance for the nuclear targeting of REST (34). Accordingly, it may be hypothesized that only REST4 maintains the ability of nuclear targeting in neuronal tissues.

Inducing factors of REST4. Recently, factors that regulate alternative splicing of REST and induce REST4 have been identified. Neural-specific Ser/Arg repeat-related protein of $100 \mathrm{kDa}$ (nSR100/SRRM4) is a transcriptional repressor of genes required for neurogenesis. Raj et al (35) demonstrated that nSR100 can directly induce alternative splicing of REST transcripts, and thus produce the REST isoform, REST4. However, in non-neural cells, REST inhibits the expression of nSR100. In addition, pioglitazone, a highly selective peroxisome proliferator-activated receptor $\gamma(\operatorname{PPAR} \gamma)$ agonist that is used to treat diabetes, has been reported to increase the expression of REST4 in HepG2 cells (36). In addition, protein kinase A, which inhibits repression of the cholinergic gene locus by REST, has been demonstrated to promote the production of REST4, whereas its inhibitor, H89, was able to suppress the expression of REST4 in PC12 cells (37).

REST4 weakens REST function. Since it lacks the C-terminal repression domain of REST and the CoREST binding domain, REST4 loses the transcriptional silencing ability of REST; however, it can suppress the silencing function of REST by inhibiting the binding of NRSF/REST to repressor element-1 (RE-1)/NRSE. This process is known as the antisilencer mechanism of gene regulation $(37,38)$. In addition, REST4 has been determined to be localized to the nucleus, and this nuclear-targeting signal exists in the zinc-finger domains (21). However, the complicated mechanism by which REST4 


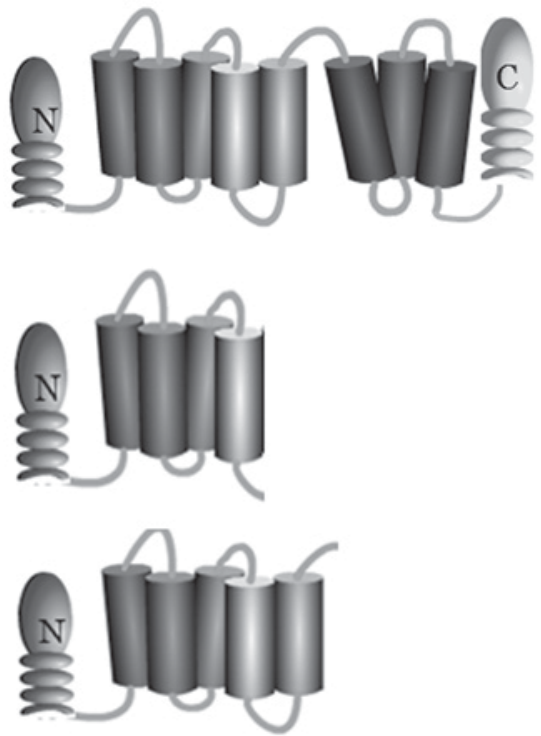

REST

REST1

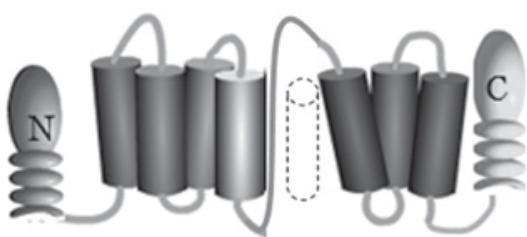

REST4

REST5

Figure 1. Protein structure of REST, REST1, REST4 and REST5. REST contains the $\mathrm{N}$-terminal repression domain, $\mathrm{C}$-terminal repression domain and number 1-8 zinc fingers. However, REST1 contains the N-terminal repression domain and number 1-4 zinc fingers, REST4 contains the $\mathrm{N}$-terminal repression domain and number 1-5 zinc fingers, and REST5 does not contain the number 5 zinc finger. REST, repressor element silencing transcription factor.
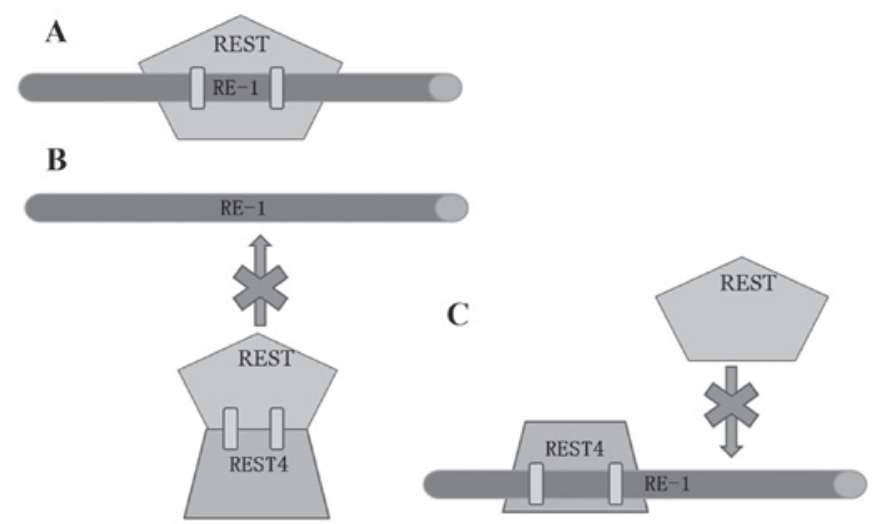

Figure 2. Relationship between RE-1, REST and REST4. (A) In norma conditions, REST can target RE-1/NRSE to serve a role in transcriptional repression. (B) REST4 may form a heterodimer with REST blocking the ability of REST to bind to RE-1/NRSE. (C) REST4 may combine with RE-1/NRSE to inhibit REST binding to RE-1/NRSE. NRSE, neuron-restrictive silencer element; RE-1, repressor element-1; REST, repressor element silencing transcription factor.

interferes with REST remains controversial (Fig. 2). Previous studies have indicated that REST4 forms a heterodimer with REST, blocking the ability of REST to bind to its DNA recognition sequence, RE-1/NRSE $(37,39)$. It has also been suggested that REST4 combines with RE-1/NRSE; however, the protein-DNA interaction is considerably weaker than that of REST, thus resulting in weakened transcriptional repression (40). Furthermore, a recent study indicated that human glioma tissues that expressed REST4 exhibited reduced REST mRNA expression compared with tissues that did not express REST4 (10). Based on these findings, the present review hypothesized that REST4 may combine with REST; therefore, REST4 may be considered a therapeutic marker that can inhibit the expression of REST, so as to prevent the proliferation of glioma cells and tumor growth. Further studies are required to validate the comprehensive cell biological processes by which REST4 affects REST.

\section{Regulation of REST expression in glioma}

Recently, HDAC inhibitors (HDACIs) have been suggested to possess therapeutic potential for patients with REST-positive medulloblastoma. In REST-positive medulloblastoma, HDACIs, such as benzamides (MS-275) and suberoylanilide hydroxamic acid, were reported to decrease REST protein expression via a post-transcriptional mechanism. With the decline in REST expression, one of its target genes, synapsin 1, was increased, thus resulting in inhibition of glioma cell growth (24).

A previous study indicated that REST alone cannot induce tumorigenesis in neural cells (41). In addition, some human medulloblastomas coexpress abnormally high levels of Myc and REST (42). Majumder demonstrated that in the Myc plus REST-expressing group, but not in the control group, tumors arose in the mouse cerebellum (43). In addition, the Wnt pathway has been reported to activate REST gene transcription by stabilizing the $\beta$-catenin protein (44); this pathway may be associated with Myc-REST-mediated medulloblastoma tumorigenesis (43).

$\beta$-transducin repeats-containing protein $(\beta-\operatorname{TrCP})$ is an E3 ubiquitin ligase, which has been reported to ubiquitinate REST, and derepress REST target genes $(23,45)$. Therefore, loss of $\beta$-TrCP expression can worsen glioma progression by reducing REST degradation and enhancing REST function. The target genes of REST in glioma have recently been verified; there were only 17 target genes extracted in cell line data and only 14 target genes in tumor samples (25), but not 24 genes, which were previously detected in three non-neuronal cells (46). In addition, the target genes were slightly different in the cell line and tumor samples (25).

In addition to the aforementioned factors, telomere repeat-binding factor 2 (TRF2) has been reported to influence the growth of glioma via the regulation of REST expression. A recent study indicated that TRF2 depletion may inhibit the proliferation and reduce the survival of glioma by activating DNA checkpoints, and rendering cells vulnerable to apoptosis. In addition, the potential underlying mechanism may be that depletion of TRF2 derepresses REST target genes resulting in cell cycle arrest and the acquisition of differentiated neuron properties, including the expression of neuronal proteins (47). Another potential mechanism suggests that depletion of TRF2 may reduce REST levels in glioma, so as to increase the levels of $\beta$-III tubulin and L1 cell adhesion molecule (L1CAM), two neuronal proteins that are REST target genes (48). However, how $\beta$-III tubulin and L1CAM influence glioma remains unknown (Fig. 3). 


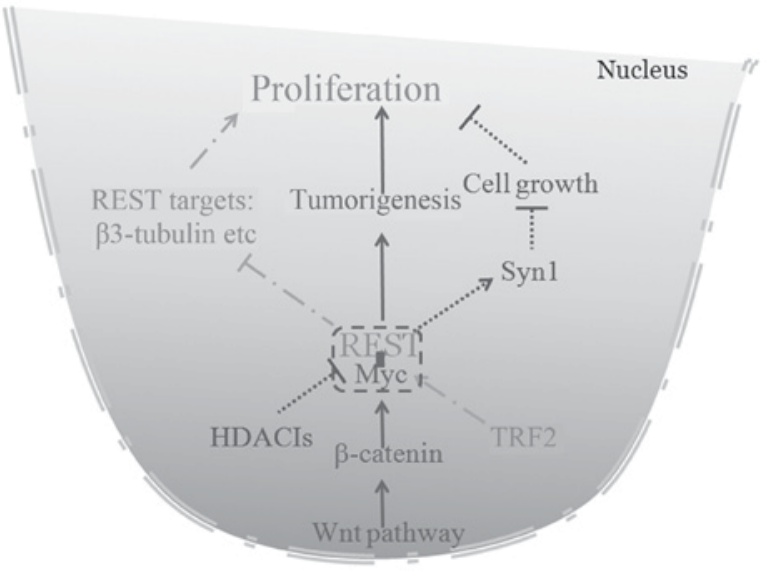

Figure 3. Regulation of REST expression, which affects glioma cell proliferation. Arrows indicate promotion or induction, whereas blunt arrows indicate inhibition or decrease. The three types of line, dotted, solid and dashed lines, represent three regulating pathways. HDACIs, histone deacetylase inhibitors; REST, repressor element silencing transcription factor; Syn1, synapsin 1; TRF2, telomere repeat-binding factor 2 .

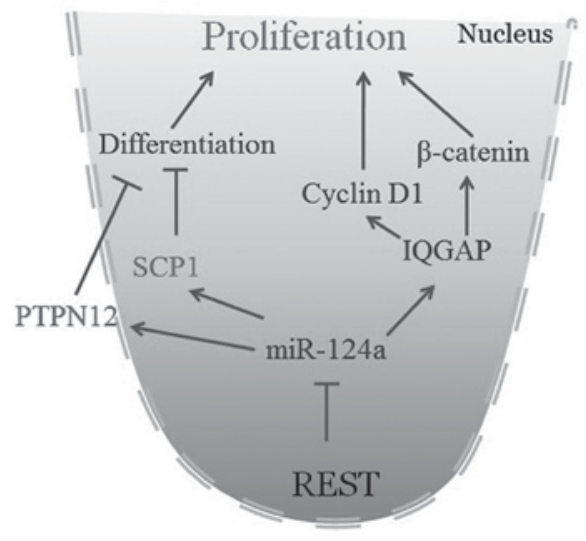

Figure 4. REST inhibits miR-124a so as to promote glioma cell proliferation in various manners. Arrows indicate promotion or induction, whereas blunt arrows indicate inhibition or decrease. IQGAP1, IQ motif containing GTPase activating protein 1; PTPN12, protein tyrosine phosphatase, non-receptor type 12; REST, repressor element silencing transcription factor; SCP1, synaptonemal complex protein 1 .

In addition to these aforementioned factors, pioglitazone, an antidiabetic drug, has also been reported to affect REST expression in glioma. When U87 cells were treated with 50 and $100 \mu \mathrm{M}$ pioglitazone, the relative expression levels of REST were significantly decreased and cell growth was inhibited (10). As a result, pioglitazone may be a potential treatment for glioma with high REST expression. When treating glioma combined with diabetes, doctors should keep in mind that pioglitazone may influence glioma progression via REST. However, this hypothesis requires further experiments to verify it in humans.

\section{Gene regulation by $\mathrm{REST}$ in glioma}

MicroRNAs (miRNAs) are essential regulators of tissue specificity (49), and REST has been reported to be associated with numerous miRNAs (50). Among them, miRNA (miR)-124a is the most representative miRNA in glioma (Fig. 4), whose overexpression is associated with improved prognosis (51). Notably, REST has been demonstrated to repress miR-124a gene expression in glioma through binding to RE-1 (52). Therefore, miR-124a levels may be decreased in high REST glioma. In addition, an increase in miR-124a expression has been reported to reduce the expression of synaptonemal complex protein 1 (SCP1) and protein tyrosine phosphatase, non-receptor type 12 (PTPN12) (9), two small phosphatases that inhibit differentiation and increase proliferation, respectively (53). Therefore, it may be concluded that REST maintains the self-renewal and tumorigenic potential of glioma cells through suppression of miR-124a and dysregulation of SCP1 and PTPN12. Furthermore, Lu et al demonstrated that the IQ motif containing GTPase activating protein 1 (IQGAP1) is a direct target of miR-124a in glioma cells. miR-124a restoration can suppress the expression of IQGAP1 and $\beta$-catenin. Furthermore, IQGAP1 suppression was able to inhibit cell proliferation and invasion by suppressing $\beta$-catenin and downstream cyclin D1 (54). Therefore, REST may inhibit glioma cell differentiation, so as to promote proliferation and invasion, via the miR-124a and IQGAP1 pathway.

\section{Conclusion}

REST is a negative regulator of genes, which exerts important functions in glioma. High REST expression has been detected in glioma, particularly in high-grade glioma. The involvement of some biological factors on the regulation of REST may explain the expression variation in glioma. The majority of studies have demonstrated that REST has a vital effect on glioma proliferation and progression. Furthermore, REST expression has been reported to be associated with glioma outcome; increased REST expression may result in shorter overall survival. Notably, REST may be considered a prognostic factor, and reducing REST expression may be a potential therapeutic strategy for the treatment of glioma.

REST4 is a splicing variant of REST that can inhibit its expression. Although the underlying mechanism remains to be elucidated, inducing REST4 does appear to decrease REST expression. Therefore, it may be hypothesized that the inducing factors of REST4, such as nSR100 and PPAR $\gamma$, may decrease the expression of REST so as to ease glioma progression and improve the prognosis of patients. However, this hypothesis requires further laboratory and clinical exploration.

However, it remains to be determined how REST impacts glioma progression and prognosis. In addition, it is unknown as to whether the REST signaling pathway may exert the same effects in glioma as is it does in non-neural cancer. The function of REST4 in glioma also remains to be elucidated, and it remains to be determined whether the expression levels of REST4, similar to REST, will influence glioma outcome. Therefore, further studies should focus on promoting clinical developments of REST and its splicing variants in glioma. In addition, the interplay between REST and REST4 in glioma remains controversial. Further in vitro and in vivo studies of REST and REST4 may identify the underlying mechanisms. In addition, further exploration is required 
to determine the complete signaling pathway of REST expression in glioma.

\section{Acknowledgements}

The present review was partially supported by the Major Project of 863 Plan (grant nos. 2012AA02A517 and 2012AA02A518), the Foundation for Open Creative Platform in Higher Education of Hunan, China (grant no. 13K002) and the National Science and Technology Major Project (grant no. 2012ZX09303013-004).

\section{References}

1. Ostrom QT, Gittleman H, Fulop J, Liu M, Blanda R, Kromer C, Wolinsky Y, Kruchko C and Barnholtz-Sloan JS: CBTRUS statistical report: Primary brain and central nervous system tumors diagnosed in the united states in 2008-2012. Neuro Oncol 17 (Suppl 4): iv1-iv62,2015.

2. Malmström A, Grønberg BH, Marosi C, Stupp R, Frappaz D, Schultz H, Abacioglu U, Tavelin B, Lhermitte B, Hegi ME, et al: Temozolomide versus standard 6-week radiotherapy versus hypofractionated radiotherapy in patients older than 60 years with glioblastoma: The Nordic randomised, phase 3 trial. Lancet Oncol 13: 916-926, 2012

3. Oike T, Suzuki Y, Sugawara K, Shirai K, Noda SE, Tamaki T, Nagaishi M, Yokoo H, Nakazato Y and Nakano T: Radiotherapy plus concomitant adjuvant temozolomide for glioblastoma: Japanese mono-institutional results. PLoS One 8: e78943, 2013.

4. Lv H, Pan G, Zheng G, Wu X, Ren H, Liu Y and Wen J: Expression and functions of the repressor element 1 (RE-1)-silencing transcription factor (REST) in breast cancer. J Cell Biochem 110 968-974, 2010

5. Reddy BY, Greco SJ, Patel PS, Trzaska KA and Rameshwar P: RE-1-silencing transcription factor shows tumor-suppressor functions and negatively regulates the oncogenic TAC1 in breast cancer cells. Proc Natl Acad Sci USA 106: 4408-4413, 2009.

6. Zhao Y, Zhu M, Yu Y, Qiu L, Zhang Y, He L and Zhang J: Brain REST/NRSF is not only a silent repressor but also an active protector. Mol Neurobiol 54: 541-550, 2017.

7. Lu T, Aron L, Zullo J, Pan Y, Kim H, Chen Y, Yang TH, Kim HM, Drake D, Liu XS, et al: REST and stress resistance in ageing and Alzheimer's disease. Nature 507: 448-454, 2014.

8. Kamal MM, Sathyan P, Singh SK, Zinn PO, Marisetty AL, Liang S, Gumin J, El-Mesallamy HO, Suki D, Colman H, et al: REST regulates oncogenic properties of glioblastoma stem cells. Stem Cells 30: 405-414, 2012

9. Conti L, Crisafulli L, Caldera V, Tortoreto M, Brilli E, Conforti P, Zunino F, Magrassi L, Schiffer D and Cattaneo E: REST controls self-renewal and tumorigenic competence of human glioblastoma cells. PLoS One 7: e38486, 2012.

10. Ren H, Gao Z, Wu N, Zeng L, Tang X, Chen X, Liu Z, Zhang W, Wang L and Li Z: Expression of REST4 in human gliomas in vivo and influence of pioglitazone on REST in vitro. Biochem Biophys Res Commun 463: 504-509, 2015.

11. Schoenherr CJ, Paquette AJ and Anderson DJ: Identification of potential target genes for the neuron-restrictive silencer factor. Proc Natl Acad Sci USA 93: 9881-9886, 1996.

12. Valouev A, Johnson DS, Sundquist A, Medina C, Anton E, Batzoglou S, Myers RM and Sidow A: Genome-wide analysis of transcription factor binding sites based on ChIP-Seq data. Nat Methods 5: 829-834, 2008.

13. Schoenherr CJ and Anderson DJ: The neuron-restrictive silencer factor (NRSF): A coordinate repressor of multiple neuron-specific genes. Science 267: 1360-1363, 1995.

14. Chong JA, Tapia-Ramírez J, Kim S, Toledo-Aral JJ, Zheng Y, Boutros MC, Altshuller YM, Frohman MA, Kraner SD and Mandel G: REST: A mammalian silencer protein that restricts sodium channel gene expression to neurons. Cell 80: 949-957, 1995.

15. Ballas N and Mandel G: The many faces of REST oversee epigenetic programming of neuronal genes. Curr Opin Neurobiol 15 : 500-506, 2005
16. Johnson R, Teh CH, Kunarso G, Wong KY, Srinivasan G, Cooper ML, Volta M, Chan SS, Lipovich L, Pollard SM, et al: REST regulates distinct transcriptional networks in embryonic and neural stem cells. PLoS Biol 6: e256, 2008.

17. Singh SK, Kagalwala MN, Parker-Thornburg J, Adams H and Majumder S: REST maintains self-renewal and pluripotency of embryonic stem cells. Nature 453: 223-227, 2008.

18. Negrini S, Prada I, D'Alessandro R and Meldolesi J: REST: An oncogene or a tumor suppressor? Trends Cell Biol 23: 289-295, 2013.

19. Fuller GN, Su X, Price RE, Cohen ZR, Lang FF, Sawaya R and Majumder S: Many human medulloblastoma tumors overexpress repressor element-1 silencing transcription (REST)/neuron-restrictive silencer factor, which can be functionally countered by REST-VP16. Mol Cancer Ther 4: 343-349, 2005.

20. Blom T, Tynninen O, Puputti M, Halonen M, Paetau A, Haapasalo H, Tanner M and Nupponen NN: Molecular genetic analysis of the REST/NRSF gene in nervous system tumors. Acta Neuropathol 112: 483-490, 2006.

21. Lepagnol-Bestel AM, Maussion G, Ramoz N, Moalic JM, Gorwood P and Simonneau M: Nrsf silencing induces molecular and subcellular changes linked to neuronal plasticity. Neuroreport 18: 441-446, 2007

22. Lee JH, Chai YG and Hersh LB: Expression patterns of mouse repressor element-1 silencing transcription factor 4 (REST4) and its possible function in neuroblastoma. J Mol Neurosci 15: 205-214, 2000.

23. Wagoner MP and Roopra A: A REST derived gene signature stratifies glioblastomas into chemotherapy resistant and responsive disease. BMC Genomics 13: 686, 2012.

24. Taylor P, Fangusaro J, Rajaram V, Goldman S, Helenowski IB, MacDonald T, Hasselblatt M, Riedemann L, Laureano A, Cooper L and Gopalakrishnan V: REST is a novel prognostic factor and therapeutic target for medulloblastoma. Mol Cancer Ther 11: 1713-1723, 2012.

25. Liang J, Tong P, Zhao W, Li Y, Zhang L, Xia Y and Yu Y: The REST gene signature predicts drug sensitivity in neuroblastoma cell lines and is significantly associated with neuroblastoma tumor stage. Int J Mol Sci 15: 11220-11233, 2014.

26. Okumura N, Yoshida H, Kitagishi Y, Nishimura Y and Matsuda S: Alternative splicings on p53, BRCA1 and PTEN genes involved in breast cancer. Biochem Biophys Res Commun 413: 395-399, 2011.

27. Barron VA and Lou H: Alternative splicing of the neurofibromatosis type I pre-mRNA. Biosci Rep 32: 131-138, 2012.

28. Klinck R, Bramard A, Inkel L, Dufresne-Martin G, Gervais-Bird J, Madden R, Paquet ER, Koh C, Venables JP, Prinos $\mathrm{P}$, et al: Multiple alternative splicing markers for ovarian cancer. Cancer Res 68: 657-663, 2008.

29. Chen M and Manley JL: Mechanisms of alternative splicing regulation: Insights from molecular and genomics approaches. Nat Rev Mol Cell Biol 10: 741-754, 2009.

30. Calarco JA, Superina S and O'Hanlon D: Regulation of vertebrate nervous system alternative splicing and development by an SR-related protein. Cell 138: 898-910, 2009.

31. Dutertre M, Sanchez G, Barbier J, Corcos L and Auboeuf D: The emerging role of pre-messenger RNA splicing in stress responses: Sending alternative messages and silent messengers. RNA Biol 8: 740-747, 2011

32. Palm K, Belluardo N, Metsis M and Timmusk T: Neuronal expression of zinc finger transcription factor REST/NRSF/XBR gene. J Neurosci 18: 1280-1296, 1998.

33. Palm K, Metsis M and Timmusk T: Neuron-specific splicing of zinc finger transcription factor REST/NRSF/XBR is frequent in neuroblastomas and conserved in human, mouse and rat. Brain Res Mol Brain Res 72: 30-39, 1999.

34. Shimojo M: Characterization of the nuclear targeting signal of REST/NRSF. Neurosci Lett 398: 161-166, 2006.

35. Raj B, O'Hanlon D, Vessey JP, Pan Q, Ray D, Buckley NJ, Miller FD and Blencowe BJ: Cross-regulation between an alternative splicing activator and a transcription repressor controls neurogenesis. Mol Cell 43: 843-850, 2011.

36. Chen GL and Miller GM: Extensive alternative splicing of the repressor element silencing transcription factor linked to cancer. PLoS One 8: e62217, 2013.

37. Shimojo M, Paquette AJ, Anderson DJ and Hersh LB: Protein kinase A regulates cholinergic gene expression in $\mathrm{PC} 12$ cells: REST4 silences the silencing activity of neuron-restrictive silencer factor/REST. Mol Cell Biol 19: 6788-6795, 1999. 
38. Tabuchi A, Yamada T, SasagawaS, Naruse Y,MoriN and Tsuda M REST4-mediated modulation of REST/NRSF-silencing function during BDNF gene promoter activation. Biochem Biophys Res Commun 290: 415-420, 2002.

39. Hersh LB and Shimojo M: Regulation of cholinergic gene expression by the neuron restrictive silencer factor/repressor element-1 silencing transcription factor. Life Sci 72: 2021-2028, 2003.

40. Lee JH, Shimojo M, Chai YG and Hersh LB: Studies on the interaction of REST4 with the cholinergic repressor element-1/neuron restrictive silencer element. Brain Res Mol Brain Res 80: 88-98, 2000.

41. Paquette AJ, Perez SE and Anderson DJ: Constitutive expression of the neuron-restrictive silencer factor (NRSF)/REST in differentiating neurons disrupts neuronal gene expression and causes axon pathfinding errors in vivo. Proc Natl Acad Sci USA 97: 12318-12323, 2000.

42. Su X, Gopalakrishnan V, Stearns D, Aldape K, Lang FF, Fuller G, Snyder E, Eberhart CG and Majumder S: Abnormal expression of REST/NRSF and Myc in neural stem/progenitor cells causes cerebellar tumors by blocking neuronal differentiation. Mol Cell Biol 26: 1666-1678, 2006.

43. Majumder S: REST in good times and bad-roles in tumor suppressor and oncogenic activities. Cell Cycle 17: 1929-1935, 2006.

44. Nishihara S, Tsuda L and Ogura T: The canonical Wnt pathway directly regulates NRSF/REST expression in chick spinal cord. Biochem Biophys Res Commun 311: 55-63, 2003.

45. Westbrook TF, Hu G, Ang XL, Mulligan P, Pavlova NN, Liang A, Leng Y, Maehr R, Shi Y, Harper JW and Elledge SJ: SCFbeta-TRCP controls oncogenic transformation and neural differentiation through REST degradation. Nature 452: 370-374, 2008.

46. Wagoner MP, Gunsalus KT, Schoenike B, Richardson AL, Friedl A and Roopra A: The transcription factor REST is lost in aggressive breast cancer. PLoS Genet 6: e1000979, 2010.
47. Bai Y, Lathia JD, Zhang P, Flavahan W, Rich JN and Mattson MP: Molecular targeting of TRF2 suppresses the growth and tumorigenesis of glioblastoma stem cells. Glia 62: 1687-1698, 2014.

48. Zhang P, Pazin MJ, Schwartz CM, Becker KG, Wersto RP, Dilley CM and Mattson MP: Nontelomeric TRF2-REST interaction modulates neuronal gene silencing and fate of tumor and stem cells. Curr Biol 18: 1489-1494, 2008.

49. Landgraf P, Rusu M, Sheridan R, Sewer A, Iovino N, Aravin A, Pfeffer S, Rice A, Kamphorst AO, Landthaler M, et al: A mammalian microRNA expression atlas based on small RNA library sequencing. Cell 129: 1401-1414, 2007.

50. Qureshi IA and Mehler MF: Regulation of non-coding RNA networks in the nervous system-what's the REST of the story? Neurosci Lett 466: 73-80, 2009.

51. Fowler A, Thomson D, Giles K, Maleki S, Mreich E, Wheeler H, Leedman P, Biggs M, Cook R, Little N, et al: miR-124a is frequently down-regulated in glioblastoma and is involved in migration and invasion. Eur J Cancer 47: 953-963, 2011.

52. Tivnan A, Zhao J, Johns TG, Day BW, Stringer BW, Boyd AW, Tiwari S, Giles KM, Teo C and McDonald KL: The tumor suppressor microRNA, miR-124a, is regulated by epigenetic silencing and by the transcriptional factor, REST in glioblastoma. Tumor Biol 35: 1459-1465, 2014.

53. Sun T, Aceto N, Meerbrey KL, Kessler JD, Zhou C, Migliaccio I, Nguyen DX, Pavlova NN, Botero M, Huang J, et al: Activation of multiple proto-oncogenic tyrosine kinases in breast cancer via loss of the PTPN12 phosphatase. Cell 144: 703-718, 2011.

54. Lu SH, Jiang XJ, Xiao GL, Liu DY and Yuan XR: miR-124a restoration inhibits glioma cell proliferation and invasion by suppressing IQGAP1 and $\beta$-catenin. Oncol Rep 32: 2104-2110, 2014. 